\title{
Keeping in close contact
}

Metal nanoparticles on graphene oxide (GO) supports are the catalytically active species in a range of heterogeneous reactions. Despite the widespread applicability of such supported systems, it is not clear if all the nanoparticles on a single GO support, or just a select few, are catalytically active. Numerous GO platelets (or particles) are used as supports in scale-up reactions but the fraction of the supported nanoparticles that are electrically 'connected' has hitherto remained unclear.

Now, writing in Angewandte Chemie International Edition,

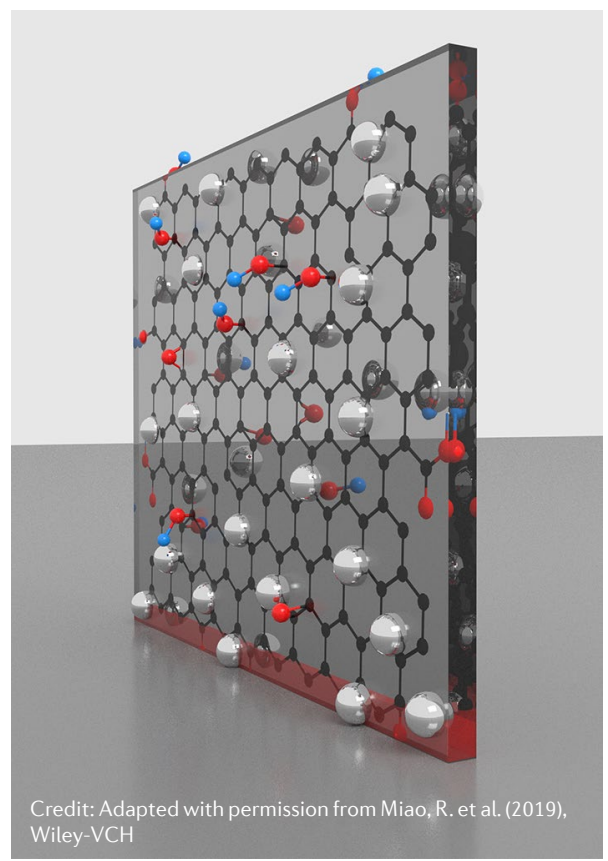

Richard Compton and colleagues investigate the hydrogen oxidation reaction (HOR) and the hydrogen evolution reaction (HER) using techniques capable of measuring the activity of single nanoparticles and reveal that only nanoparticles close to the electrical contact are active. In their experiments, platelets of GO of micrometre dimensions are decorated, in a highly disperse manner, with Pd nanoparticles with an average diameter of $2.7 \mathrm{~nm}$.

Electrochemical probing of either the HOR or HER show that only Pd nanoparticles within a very short distance of the electrical contact, between the platelet and a carbon fibre wire electrode, are catalytically active. "Indeed, most of the nanoparticles on the GO supports are not active," explains Compton. The restriction of the active area to the zone between the Pd/GO and the electrode is a consequence of the high resistance of the GO support, which is approximately $3 \mathrm{~nm}$ thick, hence comprises 2-5 layers. "The active nanoparticles are limited to those close enough to the point of contact to allow electron transfer from the electrode to the nanoparticle, possibly constrained by electron tunnelling," says Compton.

Using impact electrochemistry, the intrinsic catalytic activity of the individual Pd nanoparticles for both reactions can be studied. The arrival of a single entity of $\mathrm{Pd} / \mathrm{GO}$ at the electrode is represented by a sharp onset of current and the entity is immobilized on the electrode for around 150 seconds. This long residence time enables the study of the catalytic reaction of a single $\mathrm{Pd} / \mathrm{GO}$ entity.

Kinetics of the HOR and HER reveal that the electrochemical rates of both reactions are fast compared with the rates of diffusion of reactants to the $\mathrm{Pd} / \mathrm{GO}$ entities at the electrode. The system could be used to investigate other catalytic reactions with fast rate constants. "In addition, we wish to extend this approach to layered materials (other than GO) and investigate the extent to which the nanoparticles distributed over the surface of a catalyst support are electrochemically active at the level of a single entity of the support material," says Compton.

"The practical implication of this observation is that care is needed in moving from single entities (of catalyst support) to that of ensembles, so as to design support particles in which all the nanoparticles are electrically connected," says Compton. "Both the intrinsic conductivity of the entities (such as the particles of graphene oxide in our example) and the contact resistance between the entities in an ensemble are crucial parameters to understand and to control."

Alison Stoddart

ORIGINAL ARTICLE Miao, R. et al. Electron transfer to decorated graphene oxide particles. Angew. Chem. Int. Ed. https://doi.org/10.1002/ anie.201907393 (2019) 